\title{
28 Research Square \\ Husband/Wife Joint AB Blood Group is Associated With Clinical Outcomes of IVF/ICSI Treatment in Chinese Women
}

\section{Xiao Bao}

The First Affiliated Hospital of Zhengzhou University

Feifei Zhao

The First Affiliated Hospital of Zhengzhou University

\section{Hao Shi}

The First Affiliated Hospital of Zhengzhou University

\section{Zhiqin Bu}

The First Affiliated Hospital of Zhengzhou University

\section{Yuling Lang}

The First Affiliated Hospital of Zhengzhou University

Ying-Pu Sun ( $\square$ syp2008@vip.sina.com )

Zhengzhou University First Affiliated Hospital https://orcid.org/0000-0001-9935-9053

\section{Research}

Keywords: Husband/wife Joint ABO Blood Group, IVF/ICSI treatment, Clinical outcomes

Posted Date: August 24th, 2021

DOI: https://doi.org/10.21203/rs.3.rs-826753/v1

License: @ (1) This work is licensed under a Creative Commons Attribution 4.0 International License. Read Full License 


\section{Abstract}

Background: A number of publications have been studied the relation between blood group and female infertility including ovarian reserve, recurrent miscarriage and live birth, there is a lack of literature investigating joint wife/husband ABO blood type in large cohort. The aim of this study was to investigate the association between couple combinations for ABO blood groups and ART outcomes in patients undergoing IVF/ICSI.

Methods: This retrospective cohort study included 30,717 couples underwent IVF cycles between 2010 and 2019. Clinical outcomes of IVF treatment was the primary outcome. History of spontaneous miscarriage, embryo quality, and birth sex, weights, defects rate were also studied. Multivariable logistic regression was used to examine the association. Odds ratios (OR) with $95 \%$ confidence intervals (Cl) for each outcome were estimated.

Results: There was no difference in the baseline demographics between the blood type groups. There was a statistically significantly positive association between the combination of female blood type $A B$ and male blood type $A B$ with biochemical pregnancy, clinical pregnancy, and live birth rate (OR 1.36; 95\% Cl, 1.05-1.78; P 0.02 and OR $1.31 ; 95 \% \mathrm{Cl}, 1.03-1.68 ; \mathrm{P} 0.031$ and $\mathrm{OR} 1.28 ; 95 \% \mathrm{Cl}, 1.01-1.63 ; \mathrm{P} 0.041$ respectively). No statistically significant difference was observed between joint wife/husband $\mathrm{ABO}$ blood types and high-quality embryo rate, early abortion rate, birth sex, birth weights and birth defects rate.

Conclusions: Our findings suggest that husband/wife mating AB blood type may increase the success rate of IVF/ICSI cycles.

\section{Introduction}

ABO blood group antigen is a complex molecule expressed on the surface of human red blood cells and some other cell types and various tissues, they play major role in transfusion medicine, meanwhile, an increasing evidence suggested $A B O$ blood group is related to the development of many human diseases. A number of publications have been studied the relation between blood group and female infertility including ovarian reserve, recurrent miscarriage and live birth.

As early as 1960, some authors proposed that ABO blood incompatibility may be associated with infertility. ${ }^{(1,2)}$ In 1967 Schwimmer et al. suggested that ABO blood group incompatibility was one of the possible immunologic causes of infertility, they found that compared with couples with organic causes of infertility, couples with primary and secondary unexplained infertility possessed of higher incidence of $\mathrm{ABO}$ incompatibility. ${ }^{\left({ }^{3}\right)}$ However, the association between $A B O$ blood groups and infertility has been a point of controversy, while some studies support the absence of any relationship between blood groups and infertility in different populations. ${ }^{(4)}$ The correlation of blood groups to ovarian reserve was investigated by multiple studies. Nejat et al showed that blood type 0 was associated with diminished ovarian reserve and that the $\mathrm{A}$ blood group antigen appears to be protective of ovarian reserve. ${ }^{(5)}$ On the contrary, Lin et al ${ }^{(6)}$ showed that Chinese women with blood type $O$ had less diminished ovarian reserve than women with blood types $B$ and $A B$, who had more diminished ovarian reserve while blood type $A$ was not associated with ovarian reserve. Other studies could not confirm this and show no correlation of blood groups to ovarian reserve response during IVF treatment. ${ }^{(7-10)}$ Recently, a systematic review and meta-analysis was conducted and suggested $\mathrm{ABO}$ blood groups not associated with ovarian reserve, Ovarian hyperstimulation syndrome (OHSS) and outcomes of ART. ${ }^{(11)}$ 
Two retrospective studies on infertile women undergoing IVF that live birth relates to ABO blood type came to conflicting conclusions. ${ }^{(12,13)}$ There were also studies suggest ABO blood type has impact on female infertility by related with incidence of ovarian hyperstimulation syndrome and endometriosis, which are important influence factors of pregnancy. A predominance of blood group A was shown in patients with endometriosis by studying 231 women with endometriosis and 166 infertile women without endometriosis. ${ }^{(14)}$ One case-control study on 121 patients in Caucasians supposed a positive association between A blood group and early-onset ovarian hyperstimulation syndrome. ${ }^{(15)}$

Despite the aforementioned findings, there is a lack of literature investigating joint wife/husband ABO blood type in large cohort, we set out to explore whether ABO blood type is associated with clinical outcomes of IVF treatment, history of spontaneous miscarriage, embryo quality parameters, and birth sex, weights, defects rate in Chinese infertile couples.

\section{Materials And Methods Study Population}

This study was approved by the ethics committee of the First Affiliated Hospital of Zhengzhou University. Patients undergoing cycles of IVF/ICSI at the Reproductive Medical Center, First Affiliated Hospital of Zhengzhou University, between January 2010 and December 2019 were included in this study. 30,717 infertility couples with ABO blood groups and Rhesus factor testing, with the wife's age between 25-35 years old. Potential confounders may cause spontaneous abortion or affect ART outcomes were excluded in this study. The complete information of age, body mass index (BMI), ABO and Rh blood type, duration of subfertility, Primary subfertility, ART outcomes and birth information of each couple were collected from the Clinical Reproductive Medicine Management System/Electronic Medical Record Cohort Database of the Reproductive Medical Center of the First Affiliated Hospital of Zhengzhou University.

\section{Clinical and Laboratory Protocols}

A blood type and screen is obtained in all patients as part of the initial infertility workup at hospital's laboratory. Controlled ovarian stimulation (COS), hCG trigger, oocyte retrieval, embryo culture, and ET performed based on established protocols. ${ }^{(16)}$

In fresh ET, Patients with fresh embryo transfer were treated with gonadotropin releasing hormone $(\mathrm{GnRH})$ agonists to achieve suppression of ovulation and prevent a premature LH surge. ${ }^{(17)}$ When the patient achieved the criteria for pituitary suppression, Gonadotropin (Gonal-F, Merck, Germany; Puregon, Organon, Netherlands; Urofollitropin, Livzon, China) was used to initiate ovarian stimulation with the dose range of 75-300 IU based on the ovarian response (75-150 IU for normal or high ovarian response; 150-300 IU for reduced ovarian response). Exact dose of gonadotropin was adjusted based on age, body mass index (BMI, $\mathrm{kg} / \mathrm{m} 2$ ), antral follicle count (AFC), and $\mathrm{AMH}$ level of individual patient. hCG (Livzon) was injected the oocyte maturation trigger and was administered when at least three follicles had reached $18 \mathrm{~mm}$ and the lead follicle was $\geq 20 \mathrm{~mm}$. Oocytes were collected by transvaginal ultrasound-guided puncture at $37 \mathrm{~h}$ after $\mathrm{hCG}$ injection. Progesterone in oil was used for luteal support at a dose of $60 \mathrm{mg}$ per day after oocyte pick-up. 
Based on the condition of semen and the couples' reproductive history, fertilization was carried out with short-time insemination or ICSI insemination. ${ }^{(18)}$ The embryo morphology in the cleavage stage was observed and graded according to the Peter's standards ${ }^{(19)}$ on day 3. Day 5 blastocyst-stage embryos were graded according to the Gardner standard. ${ }^{(20)}$ The condition and the willingness of patient were comprehensively considered to decide whether to perform fresh ET or FET.

Based on the regularity of the menstrual cycle, Frozen ET cycles were divided into natural cycles and artificial cycles. For natural cycles, patients were allocated to undergo ultrasonic evaluation starting from day 8-9 of the menstrual cycle. When the diameter of the dominant follicle was $16-20 \mathrm{~mm}$, blood sample test for progesterone and LH levels was conducted to monitor ovulation. Thawing and transferring were performed 3 days for cleavagestage embryos or 5 days for blastocyst-stage embryos after ovulation. Intramuscular (im) progesterone (40 mg) starting on the day of ovulation and oral dydrogesterone $(20 \mathrm{mg})$ starting on the embryo transfer day were used for luteal support. For artificial cycles, patients began oral estradiol [2mg, Progynova; Bayer, Leverkusen, Germany] twice a day on cycle day 3. This dose was adjusted based on the endometrial thickness every 4 days. After 12-14 days, an ultrasound was performed and a serum progesterone level was determined. If no leading follicle was present, progesterone $(60 \mathrm{mg} \mathrm{im})$ and oral dydrogesterone [10 mg [this dose was changed to $20 \mathrm{mg} 2$ days later]] would be added to the regimen.

The serum hCG biochemical pregnancy tests were performed 14 days and 18 days after the embryo transfer. For patients with hCG was more than $50 \mathrm{IU} / \mathrm{L}$, transvaginal ultrasound was performed 35 days after embryo transfer.

\section{Study Variables}

Baseline demographics recorded for each patient included age (years), BMI (kg/m2), infertility diagnosis, $A B O$ blood type, and rhesus factor type. The primary outcome in this study was clinical outcomes of IVF treatment. Biochemical pregnancy was defined as an increase in the serum $\beta$-hCG concentration at 14 days after embryo transfer. Clinical pregnancy was defined as one or multiple gestational sac(s) and cardiac activity seen via ultrasound 35 days after embryo transfer. Live birth was defined as any birth event in which at least one baby is born alive. Early spontaneous miscarriage, which was defined as a pregnancy loss before 14 weeks of gestation following clinical pregnancy. Recurrent spontaneous abortion (RSA) was defined as the occurrence of at least two spontaneous losses before fetal viability.

\section{Statistical Analysis}

Association analysis were performed using custom R scripts. Results for continuous data are given as mean \pm standard deviation (SD). Results for categorical variables are given as number of cases ( $n$ ) with percentage of occurrence (\%). The proportions of the different blood types were compared by the chi-squared test. ANOVA was used for parametric data between blood type groups. Previous spontaneous abortion, embryo quality parameters, ART outcomes, birth sex, weight (log2 transformed) and defects were compared by blood type groups using Multivariable logistic regression models while adjusting for potential confounders. ORs with 95\% Cls were estimated from the model. Three models were used to test spontaneous abortion association: (1) a dosage model, which treated as recurrent miscarriage marked 1 and as 0 ; (2) a dominant model, which treated 0 as absent and more than 1 abortion as present; and (3) a continuous model to study the number of spontaneous abortion. $P<$ 0.05 was considered to be statistically significant.

\section{Results}


From the initial pool 39,477 cycles, 39,154 cycles have complete ABO and Rh blood type information with the female age $>25$ years and $<35$ years. We excluded potential confounders may cause spontaneous abortion or affect ART outcomes: chromosome abnormality $(n=976)$, congenital disease $(n=258)$, cervical incompetence $(n=$ $345)$, uterine malformation ( $n=1031)$, hypomenorrhea $(n=25)$, endometriosis or ovarian surgery history $(n=581)$, POI / oocyte maturation disorder (36), uterine fibroids $(n=216)$, endometrial lesions $(n=2)$, trophoblastic disease $(n=15)$, cervical cancer $(n=2)$, Male: azoospermia $(n=1981)$, necrozoospermia $(n=16)$, severe oligoasthenospermia $(n=2953)$. A total of 30,717 cycles met the inclusion criteria.

The distribution of these patients based on blood type was as follows: 8846 (28.80\%) females with blood type A; 3257 (10.60\%) females with blood type AB; 9712 (31.62\%) females with blood type B; and 8902(28.98\%) females with blood type O. For Rh blood type, 98 (0.032\%) females with blood type Rh-; 30,619 (99.68\%) females with blood type $\mathrm{Rh}+$; This distribution of blood types is similar to the males': 8624 (28.08\%) males with blood type A; 3257 (10.76\%) males with blood type AB; 9771 (31.81\%) males with blood type B; and 9016(29.35\%) males with blood type O. For Rh blood type, 120 (0.039\%) females with blood type Rh-; 330597 (99.61\%) females with blood type $\mathrm{Rh}+$. As shown in Fig. 1 and Supplemental Table 1, there was no difference in the distribution of blood type between infertile females and males.

Demographic and clinical characteristics of study population are shown in Table 1 and Supplemental Table 1. The women's average age was $30.0 \pm 2.83$ years. There was no difference in the age, BMI, duration of subfertility, primary subfertility by blood type in both female and male groups.

Given the small sample size of patients having Rh negative in population, especially when it was split into the four ABO blood groups, we do not include rhesus factor for downstream association test.

\section{Table 1}

Overall demographics and patients characteristics of female group stratified by blood type $(n=30,717)$

\begin{tabular}{|c|c|c|c|c|c|c|}
\hline Characteristics & All & $A(n=8846)$ & $B(n=9712)$ & $\begin{array}{l}A B(n= \\
3257)\end{array}$ & $O(n=8902)$ & $\begin{array}{l}P \\
\text { value }\end{array}$ \\
\hline Age & $30.06 \pm 2.83$ & $30.09 \pm 2.84$ & $30.06 \pm 2.85$ & $\begin{array}{l}29.98 \pm \\
2.82\end{array}$ & $30.05 \pm 2.81$ & 0.236 \\
\hline BMI & $22.83 \pm 3.22$ & $22.82 \pm 3.20$ & $22.83 \pm 3.19$ & $\begin{array}{l}22.91 \pm \\
3.36\end{array}$ & $22.82 \pm 3.20$ & 0.554 \\
\hline $\begin{array}{l}\text { Duration of } \\
\text { subfertility (m) }\end{array}$ & $48.22 \pm 33.58$ & $\begin{array}{l}48.47 \pm \\
33.31\end{array}$ & $\begin{array}{l}48.36 \pm \\
34.09\end{array}$ & $\begin{array}{l}47.68 \pm \\
32.06\end{array}$ & $\begin{array}{l}48.03 \pm \\
33.83\end{array}$ & 0.613 \\
\hline Primary subfertility & $\begin{array}{l}44.93 \% \\
(13801 / 30717)\end{array}$ & $\begin{array}{l}45.16 \% \\
(3995 / 8846)\end{array}$ & $\begin{array}{l}45.53 \% \\
(4422 / 9712)\end{array}$ & $\begin{array}{l}45.26 \% \\
(1474 \\
/ 3257)\end{array}$ & $\begin{array}{l}43.92 \% \\
(3910 / 8902)\end{array}$ & 0.145 \\
\hline
\end{tabular}

\section{History of Spontaneous Miscarriage Association Analysis}

We investigated the potential interaction between $\mathrm{ABO}$ blood group of husband/wife mating and the history of spontaneous miscarriage by three models as described before. There was a statistically significantly higher 
percentage of combination of female blood type $A B$ and male blood type 0 in couples with at least one spontaneous miscarriage history (odds ratio [OR] 1.03; 95\% confidence interval $[\mathrm{Cl}], 1-1.05 ; \mathrm{P}=0.0234$ ) than for the rest. However, no statistically significant difference was observed in Dosage and Continuous models (OR 1.01; 95\% $\mathrm{Cl}, 1-1.01 ; \mathrm{P} 0.243$ and $\mathrm{OR} 1.03 ; 95 \% \mathrm{Cl}, 1-1.06 ; \mathrm{P} 0.08$ respectively), as shown in Table 2.

\section{Table 2}

Multivariate logistic regression analyzes between history of spontaneous miscarriage and parental blood types combinations. 


\begin{tabular}{|c|c|c|c|c|c|c|c|}
\hline \multirow{2}{*}{$\begin{array}{l}\text { Female Blood } \\
\text { Type }\end{array}$} & \multirow{2}{*}{$\begin{array}{l}\text { Male } \\
\text { Blood } \\
\text { Type }\end{array}$} & \multicolumn{2}{|c|}{ Dosage model } & \multicolumn{2}{|c|}{ Dominant model } & \multicolumn{2}{|c|}{ Continuous model } \\
\hline & & OR $(95 \% \mathrm{Cl})$ & $\begin{array}{l}P \\
\text { value }\end{array}$ & OR (95\%Cl) & $\begin{array}{l}P \\
\text { value }\end{array}$ & OR (95\%Cl) & $P$ value \\
\hline$A B$ & B & $\begin{array}{l}1.00(0.99- \\
1.01)\end{array}$ & 0.874 & $\begin{array}{l}0.99(0.97- \\
1.01)\end{array}$ & 0.433 & $\begin{array}{l}0.99(0.96- \\
1.02)\end{array}$ & 0.384 \\
\hline$A B$ & A & $\begin{array}{l}1.00(0.99- \\
1.01)\end{array}$ & 0.753 & $\begin{array}{l}0.99(0.97- \\
1.01)\end{array}$ & 0.348 & $\begin{array}{l}1.00(0.97- \\
1.03)\end{array}$ & 0.843 \\
\hline$A B$ & 0 & $\begin{array}{l}1.01(1.00- \\
1.01)\end{array}$ & 0.243 & $\begin{array}{l}1.03(1.00- \\
1.05)\end{array}$ & 0.023 & $\begin{array}{l}1.03(1.00- \\
1.06)\end{array}$ & 0.080 \\
\hline$A B$ & $A B$ & $\begin{array}{l}0.99(0.98- \\
1.01)\end{array}$ & 0.304 & $\begin{array}{l}0.99(0.96- \\
1.02)\end{array}$ & 0.426 & $\begin{array}{l}0.98(0.94- \\
1.02)\end{array}$ & 0.323 \\
\hline B & B & $\begin{array}{l}1.00(0.99- \\
1.00)\end{array}$ & 0.521 & $\begin{array}{l}1.00(0.99- \\
1.01)\end{array}$ & 0.871 & $\begin{array}{l}0.99(0.97- \\
1.01)\end{array}$ & 0.438 \\
\hline B & A & $\begin{array}{l}1.00(0.99- \\
1.01)\end{array}$ & 0.966 & $\begin{array}{l}1.01(0.99- \\
1.02)\end{array}$ & 0.262 & $\begin{array}{l}1.01(0.99- \\
1.03)\end{array}$ & 0.213 \\
\hline B & 0 & $\begin{array}{l}1.00(1.00- \\
1.01)\end{array}$ & 0.443 & $\begin{array}{l}0.99(0.98- \\
1.01)\end{array}$ & 0.214 & $\begin{array}{l}0.99(0.98- \\
1.01)\end{array}$ & 0.566 \\
\hline B & $A B$ & $\begin{array}{l}1.00(0.99- \\
1.01)\end{array}$ & 0.963 & $\begin{array}{l}1.00(0.98- \\
1.03)\end{array}$ & 0.646 & $\begin{array}{l}1.00(0.98- \\
1.03)\end{array}$ & 0.802 \\
\hline$A$ & B & $\begin{array}{l}1.00(1.00- \\
1.01)\end{array}$ & 0.465 & $\begin{array}{l}1.01(0.99- \\
1.02)\end{array}$ & 0.433 & $\begin{array}{l}1.02(1.00- \\
1.04)\end{array}$ & 0.113 \\
\hline A & A & $\begin{array}{l}1.00(0.99- \\
1.00)\end{array}$ & 0.467 & $\begin{array}{l}0.99(0.98- \\
1.01)\end{array}$ & 0.355 & $\begin{array}{l}0.99(0.97- \\
1.01)\end{array}$ & 0.166 \\
\hline$A$ & 0 & $\begin{array}{l}1.00(0.99- \\
1.00)\end{array}$ & 0.525 & $\begin{array}{l}1.00(0.98- \\
1.01)\end{array}$ & 0.561 & $\begin{array}{l}0.99(0.97- \\
1.01)\end{array}$ & 0.388 \\
\hline$A$ & $A B$ & $\begin{array}{l}1.00(1.00- \\
1.01)\end{array}$ & 0.373 & $\begin{array}{l}1.01(0.99- \\
1.03)\end{array}$ & 0.308 & $\begin{array}{l}1.01(0.98- \\
1.04)\end{array}$ & 0.370 \\
\hline 0 & B & $\begin{array}{l}1.00(0.99- \\
1.01)\end{array}$ & 0.993 & $\begin{array}{l}1.00(0.99- \\
1.01)\end{array}$ & 0.910 & $\begin{array}{l}1.00(0.98- \\
1.02)\end{array}$ & 0.816 \\
\hline 0 & A & $\begin{array}{l}1.00(1.00- \\
1.01)\end{array}$ & 0.321 & $\begin{array}{l}1.00(0.99- \\
1.02)\end{array}$ & 0.676 & $\begin{array}{l}1.00(0.98- \\
1.02)\end{array}$ & 0.806 \\
\hline 0 & 0 & $\begin{array}{l}1.00(0.99- \\
1.00)\end{array}$ & 0.356 & $\begin{array}{l}1.00(0.99- \\
1.02)\end{array}$ & 0.731 & $\begin{array}{l}1.00(0.98- \\
1.02)\end{array}$ & 0.767 \\
\hline 0 & $A B$ & $\begin{array}{l}1.00(0.99- \\
1.01)\end{array}$ & 0.885 & $\begin{array}{l}0.99(0.97- \\
1.01)\end{array}$ & 0.348 & $\begin{array}{l}0.99(0.96- \\
1.02)\end{array}$ & 0.635 \\
\hline
\end{tabular}

\section{Embryo Quality parameters Association Analysis}

We also assessed the association between husband/wife blood types combinations and embryo quality parameters, including fertilization rate, cleavage rate and high-quality embryo rate, by adjusting the number of transfer embryos. The combination of female blood type $B$ and male blood type $A$ has a negative association with fertilization rate (OR $0.98 ; 95 \% \mathrm{Cl}, 0.97-1 ; P 0.01)$, while the combination of female blood type $\mathrm{O}$ and male blood type $A$ has a slightly negative association with cleavage rate (OR $1 ; 95 \% \mathrm{Cl}, 0.99-1 ; P 0.027)$. As evident in Table 3 , 
no statistically significant difference was observed between ABO blood group of husband/wife mating blood types and High-Quality Embryo Rate.

Table 3

\begin{tabular}{|c|c|c|c|c|c|c|c|}
\hline \multirow{2}{*}{$\begin{array}{l}\text { Female Blood } \\
\text { Type }\end{array}$} & \multirow{2}{*}{$\begin{array}{l}\text { Male } \\
\text { Blood } \\
\text { Type }\end{array}$} & \multicolumn{2}{|c|}{ Dosage model } & \multicolumn{2}{|c|}{ Dominant model } & \multicolumn{2}{|c|}{ Continuous mode } \\
\hline & & OR $(95 \% \mathrm{Cl})$ & $\begin{array}{l}P \\
\text { value }\end{array}$ & OR (95\%Cl) & $\begin{array}{l}P \\
\text { value }\end{array}$ & OR $(95 \% \mathrm{Cl})$ & $P$ value \\
\hline$A B$ & B & $\begin{array}{l}1.00(0.99- \\
1.01)\end{array}$ & 0.874 & $\begin{array}{l}0.99(0.97- \\
1.01)\end{array}$ & 0.433 & $\begin{array}{l}0.99(0.96- \\
1.02)\end{array}$ & 0.384 \\
\hline$A B$ & $A$ & $\begin{array}{l}1.00(0.99- \\
1.01)\end{array}$ & 0.753 & $\begin{array}{l}0.99(0.97- \\
1.01)\end{array}$ & 0.348 & $\begin{array}{l}1.00(0.97- \\
1.03)\end{array}$ & 0.843 \\
\hline$A B$ & 0 & $\begin{array}{l}1.01(1.00- \\
1.01)\end{array}$ & 0.243 & $\begin{array}{l}1.03(1.00- \\
1.05)\end{array}$ & 0.023 & $\begin{array}{l}1.03(1.00- \\
1.06)\end{array}$ & 0.080 \\
\hline$A B$ & $A B$ & $\begin{array}{l}0.99(0.98- \\
1.01)\end{array}$ & 0.304 & $\begin{array}{l}0.99(0.96- \\
1.02)\end{array}$ & 0.426 & $\begin{array}{l}0.98(0.94- \\
1.02)\end{array}$ & 0.323 \\
\hline B & $\mathrm{B}$ & $\begin{array}{l}1.00(0.99- \\
1.00)\end{array}$ & 0.521 & $\begin{array}{l}1.00(0.99- \\
1.01)\end{array}$ & 0.871 & $\begin{array}{l}0.99(0.97- \\
1.01)\end{array}$ & 0.438 \\
\hline B & $A$ & $\begin{array}{l}1.00(0.99- \\
1.01)\end{array}$ & 0.966 & $\begin{array}{l}1.01(0.99- \\
1.02)\end{array}$ & 0.262 & $\begin{array}{l}1.01(0.99- \\
1.03)\end{array}$ & 0.213 \\
\hline B & 0 & $\begin{array}{l}1.00(1.00- \\
1.01)\end{array}$ & 0.443 & $\begin{array}{l}0.99(0.98- \\
1.01)\end{array}$ & 0.214 & $\begin{array}{l}0.99(0.98- \\
1.01)\end{array}$ & 0.566 \\
\hline B & $A B$ & $\begin{array}{l}1.00(0.99- \\
1.01)\end{array}$ & 0.963 & $\begin{array}{l}1.00(0.98- \\
1.03)\end{array}$ & 0.646 & $\begin{array}{l}1.00(0.98- \\
1.03)\end{array}$ & 0.802 \\
\hline A & B & $\begin{array}{l}1.00(1.00- \\
1.01)\end{array}$ & 0.465 & $\begin{array}{l}1.01(0.99- \\
1.02)\end{array}$ & 0.433 & $\begin{array}{l}1.02(1.00- \\
1.04)\end{array}$ & 0.113 \\
\hline A & A & $\begin{array}{l}1.00(0.99- \\
1.00)\end{array}$ & 0.467 & $\begin{array}{l}0.99(0.98- \\
1.01)\end{array}$ & 0.355 & $\begin{array}{l}0.99(0.97- \\
1.01)\end{array}$ & 0.166 \\
\hline A & 0 & $\begin{array}{l}1.00(0.99- \\
1.00)\end{array}$ & 0.525 & $\begin{array}{l}1.00(0.98- \\
1.01)\end{array}$ & 0.561 & $\begin{array}{l}0.99(0.97- \\
1.01)\end{array}$ & 0.388 \\
\hline$A$ & $A B$ & $\begin{array}{l}1.00(1.00- \\
1.01)\end{array}$ & 0.373 & $\begin{array}{l}1.01(0.99- \\
1.03)\end{array}$ & 0.308 & $\begin{array}{l}1.01(0.98- \\
1.04)\end{array}$ & 0.370 \\
\hline 0 & $B$ & $\begin{array}{l}1.00(0.99- \\
1.01)\end{array}$ & 0.993 & $\begin{array}{l}1.00(0.99- \\
1.01)\end{array}$ & 0.910 & $\begin{array}{l}1.00(0.98- \\
1.02)\end{array}$ & 0.816 \\
\hline 0 & $A$ & $\begin{array}{l}1.00(1.00- \\
1.01)\end{array}$ & 0.321 & $\begin{array}{l}1.00(0.99- \\
1.02)\end{array}$ & 0.676 & $\begin{array}{l}1.00(0.98- \\
1.02)\end{array}$ & 0.806 \\
\hline 0 & 0 & $\begin{array}{l}1.00(0.99- \\
1.00)\end{array}$ & 0.356 & $\begin{array}{l}1.00(0.99- \\
1.02)\end{array}$ & 0.731 & $\begin{array}{l}1.00(0.98- \\
1.02)\end{array}$ & 0.767 \\
\hline 0 & $A B$ & $\begin{array}{l}1.00(0.99- \\
1.01)\end{array}$ & 0.885 & $\begin{array}{l}0.99(0.97- \\
1.01)\end{array}$ & 0.348 & $\begin{array}{l}0.99(0.96- \\
1.02)\end{array}$ & 0.635 \\
\hline
\end{tabular}

Table 3 
Multivariate logistic regression analyzes between embryo quality parameters and parental blood types combinations by adjusting for controlled ovarian hyperstimulation protocols. 


\begin{tabular}{|c|c|c|c|c|c|c|c|}
\hline \multirow{2}{*}{$\begin{array}{l}\text { Female } \\
\text { Blood } \\
\text { Type }\end{array}$} & \multirow{2}{*}{$\begin{array}{l}\text { Male } \\
\text { Blood } \\
\text { Type }\end{array}$} & \multicolumn{2}{|c|}{ Fertilization Rate } & \multicolumn{2}{|c|}{ Cleavage Rate } & \multicolumn{2}{|c|}{ High Quality Embryo Rate } \\
\hline & & OR $(95 \% \mathrm{Cl})$ & $\begin{array}{l}P \\
\text { value }\end{array}$ & $\begin{array}{l}\text { OR } \\
(95 \% \mathrm{Cl})\end{array}$ & $\begin{array}{l}P \\
\text { value }\end{array}$ & OR $(95 \% \mathrm{Cl})$ & $P$ value \\
\hline B & $A$ & $\begin{array}{l}0.98(0.97- \\
1.00)\end{array}$ & 0.010 & $\begin{array}{l}1.00(1.00- \\
1.01)\end{array}$ & 0.251 & $\begin{array}{l}0.99(0.97- \\
1.01)\end{array}$ & 0.354 \\
\hline B & 0 & $\begin{array}{l}1.01(0.99- \\
1.02)\end{array}$ & 0.245 & $\begin{array}{l}1.00 \\
(1.00- \\
1.00)\end{array}$ & 0.942 & $\begin{array}{l}1.02(1.00- \\
1.04)\end{array}$ & 0.110 \\
\hline B & B & $\begin{array}{l}1.01(0.99- \\
1.02)\end{array}$ & 0.315 & $\begin{array}{l}1.00 \\
(1.00- \\
1.00)\end{array}$ & 0.783 & $\begin{array}{l}0.99(0.97- \\
1.01)\end{array}$ & 0.442 \\
\hline B & $A B$ & $\begin{array}{l}1.01(0.98- \\
1.03)\end{array}$ & 0.563 & $\begin{array}{l}1.00(0.99- \\
1.00)\end{array}$ & 0.254 & $\begin{array}{l}1.00 \\
(0.97- \\
1.03)\end{array}$ & 0.843 \\
\hline$A B$ & $A$ & $\begin{array}{l}1.00(0.98- \\
1.02)\end{array}$ & 0.813 & $\begin{array}{l}1.00(1.00- \\
1.01)\end{array}$ & 0.343 & $\begin{array}{l}0.99(0.96- \\
1.02)\end{array}$ & 0.564 \\
\hline$A B$ & 0 & $\begin{array}{l}1.00(0.98- \\
1.03)\end{array}$ & 0.854 & $\begin{array}{l}1.00(0.99- \\
1.00)\end{array}$ & 0.386 & $\begin{array}{l}1.01(0.98- \\
1.04)\end{array}$ & 0.520 \\
\hline$A B$ & B & $\begin{array}{l}1.00(0.98- \\
1.02)\end{array}$ & 0.847 & $\begin{array}{l}1.00 \\
(0.99- \\
1.01)\end{array}$ & 0.818 & $\begin{array}{l}1.01(0.98- \\
1.04)\end{array}$ & 0.529 \\
\hline$A B$ & $A B$ & $\begin{array}{l}1.01(0.97- \\
1.04)\end{array}$ & 0.701 & $\begin{array}{l}1.00 \\
(0.99- \\
1.01)\end{array}$ & 0.832 & $\begin{array}{l}0.98(0.94- \\
1.02)\end{array}$ & 0.313 \\
\hline A & A & $\begin{array}{l}1.01(0.99- \\
1.02)\end{array}$ & 0.278 & $\begin{array}{l}1.00(1.00- \\
1.01)\end{array}$ & 0.654 & $\begin{array}{l}1.01(0.99- \\
1.03)\end{array}$ & 0.510 \\
\hline$A$ & 0 & $\begin{array}{l}1.00(0.99- \\
1.02)\end{array}$ & 0.839 & $\begin{array}{l}1.00(0.99- \\
1.00)\end{array}$ & 0.464 & $\begin{array}{l}0.98(0.96- \\
1.00)\end{array}$ & 0.061 \\
\hline A & B & $\begin{array}{l}0.99(0.98- \\
1.01)\end{array}$ & 0.282 & $\begin{array}{l}1.00 \\
(1.00- \\
1.00)\end{array}$ & 0.992 & $\begin{array}{l}1.01(0.99- \\
1.03)\end{array}$ & 0.308 \\
\hline A & $A B$ & $\begin{array}{l}1.00(0.97- \\
1.02)\end{array}$ & 0.782 & $\begin{array}{l}1.00(1.00- \\
1.01)\end{array}$ & 0.658 & $\begin{array}{l}1.00 \\
(0.97- \\
1.03)\end{array}$ & 0.816 \\
\hline 0 & $A$ & $\begin{array}{l}1.01(1.00- \\
1.03)\end{array}$ & 0.089 & $\begin{array}{l}1.00(0.99- \\
1.00)\end{array}$ & 0.027 & $\begin{array}{l}1.01(0.99- \\
1.03)\end{array}$ & 0.497 \\
\hline 0 & 0 & $\begin{array}{l}0.99(0.97- \\
1.00)\end{array}$ & 0.127 & $\begin{array}{l}1.00(1.00- \\
1.01)\end{array}$ & 0.171 & $\begin{array}{l}1.00 \\
(0.98- \\
1.02)\end{array}$ & 0.860 \\
\hline 0 & B & $\begin{array}{l}1.00(0.99- \\
1.02)\end{array}$ & 0.840 & $\begin{array}{l}1.00 \\
(1.00- \\
1.00)\end{array}$ & 0.680 & $\begin{array}{l}0.99(0.98- \\
1.01)\end{array}$ & 0.508 \\
\hline 0 & $A B$ & $\begin{array}{l}0.99(0.97- \\
1.02)\end{array}$ & 0.565 & $\begin{array}{l}1.00(1.00- \\
1.01)\end{array}$ & 0.559 & $\begin{array}{l}1.00 \\
(0.98- \\
1.03)\end{array}$ & 0.808 \\
\hline
\end{tabular}




\section{ART Outcomes Association Analysis}

We have further compared the outcomes of treatment to blood groups, including Biochemical and clinical Pregnancy, early abortion rate, live birth rate. As shown in Table 4, there was a statistically significantly positive association between the combination of female blood type $A B$ and male blood type $A B$ with biochemical pregnancy, clinical pregnancy, and live birth rate (OR 1.36; 95\% Cl, 1.05-1.78; P 0.02 and OR $1.31 ; 95 \% \mathrm{Cl}, 1.03-$ $1.68 ; \mathrm{P} 0.031$ and $\mathrm{OR} 1.28 ; 95 \% \mathrm{Cl}, 1.01-1.63 ; \mathrm{P} 0.041$ respectively). No statistically significant difference was observed between couple combinations for $\mathrm{ABO}$ blood groups and early abortion rate. 
Table 4

\begin{tabular}{|c|c|c|c|c|c|c|c|}
\hline \multirow{2}{*}{$\begin{array}{l}\text { Female } \\
\text { Blood } \\
\text { Type }\end{array}$} & \multirow{2}{*}{$\begin{array}{l}\text { Male } \\
\text { Blood } \\
\text { Type }\end{array}$} & \multicolumn{2}{|c|}{ Fertilization Rate } & \multicolumn{2}{|c|}{ Cleavage Rate } & \multicolumn{2}{|c|}{ High Quality Embryo Rate } \\
\hline & & OR $(95 \% \mathrm{Cl})$ & $\begin{array}{l}P \\
\text { value }\end{array}$ & $\begin{array}{l}\text { OR } \\
(95 \% \mathrm{Cl})\end{array}$ & $\begin{array}{l}P \\
\text { value }\end{array}$ & OR $(95 \% \mathrm{Cl})$ & $P$ value \\
\hline B & A & $\begin{array}{l}0.98(0.97- \\
1.00)\end{array}$ & 0.010 & $\begin{array}{l}1.00(1.00- \\
1.01)\end{array}$ & 0.251 & $\begin{array}{l}0.99(0.97- \\
1.01)\end{array}$ & 0.354 \\
\hline B & 0 & $\begin{array}{l}1.01(0.99- \\
1.02)\end{array}$ & 0.245 & $\begin{array}{l}1.00 \\
(1.00- \\
1.00)\end{array}$ & 0.942 & $\begin{array}{l}1.02(1.00- \\
1.04)\end{array}$ & 0.110 \\
\hline B & B & $\begin{array}{l}1.01(0.99- \\
1.02)\end{array}$ & 0.315 & $\begin{array}{l}1.00 \\
(1.00- \\
1.00)\end{array}$ & 0.783 & $\begin{array}{l}0.99(0.97- \\
1.01)\end{array}$ & 0.442 \\
\hline B & $A B$ & $\begin{array}{l}1.01(0.98- \\
1.03)\end{array}$ & 0.563 & $\begin{array}{l}1.00(0.99- \\
1.00)\end{array}$ & 0.254 & $\begin{array}{l}1.00 \\
(0.97- \\
1.03)\end{array}$ & 0.843 \\
\hline$A B$ & A & $\begin{array}{l}1.00(0.98- \\
1.02)\end{array}$ & 0.813 & $\begin{array}{l}1.00(1.00- \\
1.01)\end{array}$ & 0.343 & $\begin{array}{l}0.99(0.96- \\
1.02)\end{array}$ & 0.564 \\
\hline$A B$ & 0 & $\begin{array}{l}1.00(0.98- \\
1.03)\end{array}$ & 0.854 & $\begin{array}{l}1.00(0.99- \\
1.00)\end{array}$ & 0.386 & $\begin{array}{l}1.01(0.98- \\
1.04)\end{array}$ & 0.520 \\
\hline$A B$ & B & $\begin{array}{l}1.00(0.98- \\
1.02)\end{array}$ & 0.847 & $\begin{array}{l}1.00 \\
(0.99- \\
1.01)\end{array}$ & 0.818 & $\begin{array}{l}1.01(0.98- \\
1.04)\end{array}$ & 0.529 \\
\hline$A B$ & $A B$ & $\begin{array}{l}1.01(0.97- \\
1.04)\end{array}$ & 0.701 & $\begin{array}{l}1.00 \\
(0.99- \\
1.01)\end{array}$ & 0.832 & $\begin{array}{l}0.98(0.94- \\
1.02)\end{array}$ & 0.313 \\
\hline A & A & $\begin{array}{l}1.01(0.99- \\
1.02)\end{array}$ & 0.278 & $\begin{array}{l}1.00(1.00- \\
1.01)\end{array}$ & 0.654 & $\begin{array}{l}1.01(0.99- \\
1.03)\end{array}$ & 0.510 \\
\hline A & 0 & $\begin{array}{l}1.00(0.99- \\
1.02)\end{array}$ & 0.839 & $\begin{array}{l}1.00(0.99- \\
1.00)\end{array}$ & 0.464 & $\begin{array}{l}0.98(0.96- \\
1.00)\end{array}$ & 0.061 \\
\hline A & B & $\begin{array}{l}0.99(0.98- \\
1.01)\end{array}$ & 0.282 & $\begin{array}{l}1.00 \\
(1.00- \\
1.00)\end{array}$ & 0.992 & $\begin{array}{l}1.01(0.99- \\
1.03)\end{array}$ & 0.308 \\
\hline A & $A B$ & $\begin{array}{l}1.00(0.97- \\
1.02)\end{array}$ & 0.782 & $\begin{array}{l}1.00(1.00- \\
1.01)\end{array}$ & 0.658 & $\begin{array}{l}1.00 \\
(0.97- \\
1.03)\end{array}$ & 0.816 \\
\hline 0 & A & $\begin{array}{l}1.01(1.00- \\
1.03)\end{array}$ & 0.089 & $\begin{array}{l}1.00(0.99- \\
1.00)\end{array}$ & 0.027 & $\begin{array}{l}1.01(0.99- \\
1.03)\end{array}$ & 0.497 \\
\hline 0 & 0 & $\begin{array}{l}0.99(0.97- \\
1.00)\end{array}$ & 0.127 & $\begin{array}{l}1.00(1.00- \\
1.01)\end{array}$ & 0.171 & $\begin{array}{l}1.00 \\
(0.98- \\
1.02)\end{array}$ & 0.860 \\
\hline 0 & B & $\begin{array}{l}1.00(0.99- \\
1.02)\end{array}$ & 0.840 & $\begin{array}{l}1.00 \\
(1.00- \\
1.00)\end{array}$ & 0.680 & $\begin{array}{l}0.99(0.98- \\
1.01)\end{array}$ & 0.508 \\
\hline
\end{tabular}




\begin{tabular}{|c|c|c|c|c|c|c|c|}
\hline \multirow{2}{*}{$\begin{array}{l}\text { Female } \\
\text { Blood } \\
\text { Type }\end{array}$} & \multirow{2}{*}{$\begin{array}{l}\text { Male } \\
\text { Blood } \\
\text { Type }\end{array}$} & \multicolumn{2}{|c|}{ Fertilization Rate } & \multicolumn{2}{|c|}{ Cleavage Rate } & \multicolumn{2}{|c|}{ High Quality Embryo Rate } \\
\hline & & OR $(95 \% \mathrm{Cl})$ & $\begin{array}{l}P \\
\text { value }\end{array}$ & $\begin{array}{l}\text { OR } \\
(95 \% \mathrm{Cl})\end{array}$ & $\begin{array}{l}P \\
\text { value }\end{array}$ & OR (95\%Cl) & $P$ value \\
\hline 0 & $A B$ & $\begin{array}{l}0.99(0.97- \\
1.02)\end{array}$ & 0.565 & $\begin{array}{l}1.00 \text { (1.00- } \\
1.01)\end{array}$ & 0.559 & $\begin{array}{l}1.00 \\
(0.98- \\
1.03)\end{array}$ & 0.808 \\
\hline
\end{tabular}

\section{Table 4}

Multivariate logistic regression analyses between ART outcomes and parental blood types combinations with adjusting for number of transfer embryo(s). 


\begin{tabular}{|c|c|c|c|c|c|c|c|c|c|}
\hline \multirow{2}{*}{$\begin{array}{l}\text { Female } \\
\text { Blood } \\
\text { Type }\end{array}$} & \multirow{2}{*}{$\begin{array}{l}\text { Male } \\
\text { Blood } \\
\text { Type }\end{array}$} & \multicolumn{2}{|l|}{$\begin{array}{l}\text { Biochemical } \\
\text { Pregnancy }\end{array}$} & \multicolumn{2}{|c|}{ Clinical pregnancy } & \multicolumn{3}{|c|}{ Early Abortion Rate } & $\begin{array}{l}\text { Live Birth } \\
\text { Rate }\end{array}$ \\
\hline & & $\begin{array}{l}\text { OR } \\
(95 \% \mathrm{Cl})\end{array}$ & $\begin{array}{l}P \\
\text { value }\end{array}$ & $\begin{array}{l}\text { OR } \\
(95 \% \mathrm{Cl})\end{array}$ & $\begin{array}{l}P \\
\text { value }\end{array}$ & $\begin{array}{l}\text { OR } \\
(95 \% \mathrm{Cl})\end{array}$ & $\begin{array}{l}P \\
\text { value }\end{array}$ & $\begin{array}{l}\text { OR } \\
(95 \% \mathrm{Cl})\end{array}$ & $\begin{array}{l}P \\
\text { value }\end{array}$ \\
\hline$A B$ & $\mathrm{~B}$ & $\begin{array}{l}1.04(0.88- \\
1.23)\end{array}$ & 0.614 & $\begin{array}{l}0.93(0.79- \\
1.09)\end{array}$ & 0.374 & $\begin{array}{l}0.87(0.69- \\
1.10)\end{array}$ & 0.255 & $\begin{array}{l}0.91(0.78- \\
1.06)\end{array}$ & 0.234 \\
\hline$A B$ & $A$ & $\begin{array}{l}0.92(0.78- \\
1.10)\end{array}$ & 0.361 & $\begin{array}{l}0.98(0.83- \\
1.16)\end{array}$ & 0.824 & $\begin{array}{l}1.08(0.87- \\
1.35)\end{array}$ & 0.479 & $\begin{array}{l}0.96(0.82- \\
1.13)\end{array}$ & 0.646 \\
\hline$A B$ & 0 & $\begin{array}{l}0.90(0.76- \\
1.07)\end{array}$ & 0.239 & $\begin{array}{l}0.97(0.83- \\
1.14)\end{array}$ & 0.742 & $\begin{array}{l}1.04(0.83- \\
1.30)\end{array}$ & 0.708 & $\begin{array}{l}1.02(0.87- \\
1.20)\end{array}$ & 0.769 \\
\hline$A B$ & $A B$ & $\begin{array}{l}1.36(1.05- \\
1.78)\end{array}$ & 0.020 & $\begin{array}{l}1.31(1.03- \\
1.68)\end{array}$ & 0.031 & $\begin{array}{l}1.01(0.71- \\
1.42)\end{array}$ & 0.975 & $\begin{array}{l}1.28(1.01- \\
1.63)\end{array}$ & 0.041 \\
\hline B & $\mathrm{B}$ & $\begin{array}{l}0.99(0.89- \\
1.10)\end{array}$ & 0.841 & $\begin{array}{l}0.98(0.88- \\
1.09)\end{array}$ & 0.749 & $\begin{array}{l}1.19(0.94- \\
1.50)\end{array}$ & 0.155 & $\begin{array}{l}0.99(0.90- \\
1.10)\end{array}$ & 0.920 \\
\hline B & $A$ & $\begin{array}{l}0.97(0.86- \\
1.09)\end{array}$ & 0.585 & $\begin{array}{l}1.00(0.90- \\
1.11)\end{array}$ & 0.972 & $\begin{array}{l}0.83(0.66- \\
1.05)\end{array}$ & 0.119 & $\begin{array}{l}0.99(0.89- \\
1.10)\end{array}$ & 0.809 \\
\hline B & 0 & $\begin{array}{l}1.06(0.95- \\
1.19)\end{array}$ & 0.305 & $\begin{array}{l}1.05(0.94- \\
1.17)\end{array}$ & 0.383 & $\begin{array}{l}0.96(0.76- \\
1.21)\end{array}$ & 0.738 & $\begin{array}{l}1.07(0.96- \\
1.19)\end{array}$ & 0.222 \\
\hline B & $A B$ & $\begin{array}{l}0.97(0.82- \\
1.14)\end{array}$ & 0.678 & $\begin{array}{l}0.94(0.80- \\
1.10)\end{array}$ & 0.458 & $\begin{array}{l}1.17(0.81- \\
1.65)\end{array}$ & 0.395 & $\begin{array}{l}0.90(0.77- \\
1.05)\end{array}$ & 0.196 \\
\hline A & $\mathrm{B}$ & $\begin{array}{l}0.99(0.88- \\
1.11)\end{array}$ & 0.861 & $\begin{array}{l}1.03(0.93- \\
1.15)\end{array}$ & 0.572 & $\begin{array}{l}1.01(0.71- \\
1.42)\end{array}$ & 0.959 & $\begin{array}{l}1.01(0.91- \\
1.13)\end{array}$ & 0.802 \\
\hline$A$ & $A$ & $\begin{array}{l}1.05(0.93- \\
1.18)\end{array}$ & 0.435 & $\begin{array}{l}0.99(0.88- \\
1.10)\end{array}$ & 0.806 & $\begin{array}{l}1.13(0.81- \\
1.56)\end{array}$ & 0.477 & $\begin{array}{l}1.03(0.93- \\
1.15)\end{array}$ & 0.556 \\
\hline A & 0 & $\begin{array}{l}0.97(0.86- \\
1.08)\end{array}$ & 0.550 & $\begin{array}{l}0.96(0.86- \\
1.08)\end{array}$ & 0.515 & $\begin{array}{l}0.82(0.58- \\
1.16)\end{array}$ & 0.275 & $\begin{array}{l}0.92(0.83- \\
1.03)\end{array}$ & 0.137 \\
\hline A & $A B$ & $\begin{array}{l}1.00(0.84- \\
1.19)\end{array}$ & 0.981 & $\begin{array}{l}1.04(0.88- \\
1.23)\end{array}$ & 0.623 & $\begin{array}{l}1.15(0.68- \\
1.86)\end{array}$ & 0.593 & $\begin{array}{l}1.09(0.92- \\
1.28)\end{array}$ & 0.315 \\
\hline 0 & $\mathrm{~B}$ & $\begin{array}{l}1.00(0.90- \\
1.12)\end{array}$ & 0.962 & $\begin{array}{l}1.02(0.92- \\
1.14)\end{array}$ & 0.690 & $\begin{array}{l}0.97(0.76- \\
1.23)\end{array}$ & 0.781 & $\begin{array}{l}1.04(0.93- \\
1.15)\end{array}$ & 0.500 \\
\hline 0 & $A$ & $\begin{array}{l}1.03(0.91- \\
1.15)\end{array}$ & 0.680 & $\begin{array}{l}1.02(0.92- \\
1.15)\end{array}$ & 0.675 & $\begin{array}{l}1.04(0.83- \\
1.31)\end{array}$ & 0.714 & $\begin{array}{l}1.00(0.89- \\
1.11)\end{array}$ & 0.971 \\
\hline 0 & 0 & $\begin{array}{l}1.02(0.91- \\
1.14)\end{array}$ & 0.739 & $\begin{array}{l}1.00(0.89- \\
1.11)\end{array}$ & 0.966 & $\begin{array}{l}1.08(0.86- \\
1.37)\end{array}$ & 0.490 & $\begin{array}{l}1.00(0.9- \\
1.120)\end{array}$ & 0.980 \\
\hline 0 & $A B$ & $\begin{array}{l}0.90(0.77- \\
1.07)\end{array}$ & 0.242 & $\begin{array}{l}0.91(0.77- \\
1.07)\end{array}$ & 0.240 & $\begin{array}{l}0.78(0.53- \\
1.13)\end{array}$ & 0.201 & $\begin{array}{l}0.92(0.79- \\
1.08)\end{array}$ & 0.303 \\
\hline
\end{tabular}

\section{Birth Sex, Weights, Defects Rate Association Analysis}

Finally, multivariate logistic regression analysis was performed to determine the relationship between blood type and birth sex, birth weights and birth defects rate after adjusting for the number of live births. As shown in Table 5, no blood type was statistically significantly associated with birth sex, birth weights and birth defects rate. 
Table 5

\begin{tabular}{|c|c|c|c|c|c|c|c|c|c|}
\hline \multirow{2}{*}{$\begin{array}{l}\text { Female } \\
\text { Blood } \\
\text { Type }\end{array}$} & \multirow{2}{*}{$\begin{array}{l}\text { Male } \\
\text { Blood } \\
\text { Type }\end{array}$} & \multicolumn{2}{|l|}{$\begin{array}{l}\text { Biochemical } \\
\text { Pregnancy }\end{array}$} & \multicolumn{2}{|c|}{ Clinical pregnancy } & \multicolumn{2}{|c|}{ Early Abortion Rate } & $\begin{array}{l}\text { Live } \\
\text { Rate }\end{array}$ & $\begin{array}{l}\text { Live Birth } \\
\text { Rate }\end{array}$ \\
\hline & & $\begin{array}{l}\text { OR } \\
(95 \% \mathrm{Cl})\end{array}$ & $\begin{array}{l}\mathrm{P} \\
\text { value }\end{array}$ & $\begin{array}{l}\text { OR } \\
(95 \% \mathrm{Cl})\end{array}$ & $\begin{array}{l}\mathrm{P} \\
\text { value }\end{array}$ & $\begin{array}{l}\text { OR } \\
(95 \% \mathrm{Cl})\end{array}$ & $\begin{array}{l}P \\
\text { value }\end{array}$ & $\begin{array}{l}\text { OR } \\
(95 \% \mathrm{Cl})\end{array}$ & $\begin{array}{l}P \\
\text { value }\end{array}$ \\
\hline$A B$ & B & $\begin{array}{l}1.04(0.88- \\
1.23)\end{array}$ & 0.614 & $\begin{array}{l}0.93(0.79- \\
1.09)\end{array}$ & 0.374 & $\begin{array}{l}0.87(0.69- \\
1.10)\end{array}$ & 0.255 & $\begin{array}{l}0.91(0.78- \\
1.06)\end{array}$ & 0.234 \\
\hline$A B$ & $A$ & $\begin{array}{l}0.92(0.78- \\
1.10)\end{array}$ & 0.361 & $\begin{array}{l}0.98(0.83- \\
1.16)\end{array}$ & 0.824 & $\begin{array}{l}1.08(0.87- \\
1.35)\end{array}$ & 0.479 & $\begin{array}{l}0.96(0.82- \\
1.13)\end{array}$ & 0.646 \\
\hline$A B$ & 0 & $\begin{array}{l}0.90(0.76- \\
1.07)\end{array}$ & 0.239 & $\begin{array}{l}0.97(0.83- \\
1.14)\end{array}$ & 0.742 & $\begin{array}{l}1.04(0.83- \\
1.30)\end{array}$ & 0.708 & $\begin{array}{l}1.02(0.87- \\
1.20)\end{array}$ & 0.769 \\
\hline$A B$ & $A B$ & $\begin{array}{l}1.36(1.05- \\
1.78)\end{array}$ & 0.020 & $\begin{array}{l}1.31(1.03- \\
1.68)\end{array}$ & 0.031 & $\begin{array}{l}1.01(0.71- \\
1.42)\end{array}$ & 0.975 & $\begin{array}{l}1.28(1.01- \\
1.63)\end{array}$ & 0.041 \\
\hline B & $\mathrm{B}$ & $\begin{array}{l}0.99(0.89- \\
1.10)\end{array}$ & 0.841 & $\begin{array}{l}0.98(0.88- \\
1.09)\end{array}$ & 0.749 & $\begin{array}{l}1.19(0.94- \\
1.50)\end{array}$ & 0.155 & $\begin{array}{l}0.99(0.90- \\
1.10)\end{array}$ & 0.920 \\
\hline B & $A$ & $\begin{array}{l}0.97(0.86- \\
1.09)\end{array}$ & 0.585 & $\begin{array}{l}1.00(0.90- \\
1.11)\end{array}$ & 0.972 & $\begin{array}{l}0.83(0.66- \\
1.05)\end{array}$ & 0.119 & $\begin{array}{l}0.99(0.89- \\
1.10)\end{array}$ & 0.809 \\
\hline B & 0 & $\begin{array}{l}1.06(0.95- \\
1.19)\end{array}$ & 0.305 & $\begin{array}{l}1.05(0.94- \\
1.17)\end{array}$ & 0.383 & $\begin{array}{l}0.96(0.76- \\
1.21)\end{array}$ & 0.738 & $\begin{array}{l}1.07(0.96- \\
1.19)\end{array}$ & 0.222 \\
\hline B & $A B$ & $\begin{array}{l}0.97(0.82- \\
1.14)\end{array}$ & 0.678 & $\begin{array}{l}0.94(0.80- \\
1.10)\end{array}$ & 0.458 & $\begin{array}{l}1.17(0.81- \\
1.65)\end{array}$ & 0.395 & $\begin{array}{l}0.90(0.77- \\
1.05)\end{array}$ & 0.196 \\
\hline A & B & $\begin{array}{l}0.99(0.88- \\
1.11)\end{array}$ & 0.861 & $\begin{array}{l}1.03(0.93- \\
1.15)\end{array}$ & 0.572 & $\begin{array}{l}1.01(0.71- \\
1.42)\end{array}$ & 0.959 & $\begin{array}{l}1.01(0.91- \\
1.13)\end{array}$ & 0.802 \\
\hline A & $A$ & $\begin{array}{l}1.05(0.93- \\
1.18)\end{array}$ & 0.435 & $\begin{array}{l}0.99(0.88- \\
1.10)\end{array}$ & 0.806 & $\begin{array}{l}1.13(0.81- \\
1.56)\end{array}$ & 0.477 & $\begin{array}{l}1.03(0.93- \\
1.15)\end{array}$ & 0.556 \\
\hline A & 0 & $\begin{array}{l}0.97(0.86- \\
1.08)\end{array}$ & 0.550 & $\begin{array}{l}0.96(0.86- \\
1.08)\end{array}$ & 0.515 & $\begin{array}{l}0.82(0.58- \\
1.16)\end{array}$ & 0.275 & $\begin{array}{l}0.92(0.83- \\
1.03)\end{array}$ & 0.137 \\
\hline$A$ & $A B$ & $\begin{array}{l}1.00(0.84- \\
1.19)\end{array}$ & 0.981 & $\begin{array}{l}1.04(0.88- \\
1.23)\end{array}$ & 0.623 & $\begin{array}{l}1.15(0.68- \\
1.86)\end{array}$ & 0.593 & $\begin{array}{l}1.09(0.92- \\
1.28)\end{array}$ & 0.315 \\
\hline 0 & $\mathrm{~B}$ & $\begin{array}{l}1.00(0.90- \\
1.12)\end{array}$ & 0.962 & $\begin{array}{l}1.02(0.92- \\
1.14)\end{array}$ & 0.690 & $\begin{array}{l}0.97(0.76- \\
1.23)\end{array}$ & 0.781 & $\begin{array}{l}1.04(0.93- \\
1.15)\end{array}$ & 0.500 \\
\hline 0 & $A$ & $\begin{array}{l}1.03(0.91- \\
1.15)\end{array}$ & 0.680 & $\begin{array}{l}1.02(0.92- \\
1.15)\end{array}$ & 0.675 & $\begin{array}{l}1.04(0.83- \\
1.31)\end{array}$ & 0.714 & $\begin{array}{l}1.00(0.89- \\
1.11)\end{array}$ & 0.971 \\
\hline 0 & 0 & $\begin{array}{l}1.02(0.91- \\
1.14)\end{array}$ & 0.739 & $\begin{array}{l}1.00(0.89- \\
1.11)\end{array}$ & 0.966 & $\begin{array}{l}1.08(0.86- \\
1.37)\end{array}$ & 0.490 & $\begin{array}{l}1.00(0.9- \\
1.120)\end{array}$ & 0.980 \\
\hline 0 & $A B$ & $\begin{array}{l}0.90(0.77- \\
1.07)\end{array}$ & 0.242 & $\begin{array}{l}0.91(0.77- \\
1.07)\end{array}$ & 0.240 & $\begin{array}{l}0.78(0.53- \\
1.13)\end{array}$ & 0.201 & $\begin{array}{l}0.92(0.79- \\
1.08)\end{array}$ & 0.303 \\
\hline
\end{tabular}

\section{Discussion}

To our known, our study represents the largest retrospective cohort study evaluating the association between wife/husband ABO blood group pairs and ART outcomes published so far, the result suggests there was a 
statistically significantly positive association between the combination of female blood type $A B$ and male blood type $A B$ with biochemical pregnancy, clinical pregnancy, and live birth rate. No statistically significant difference was observed between couple combinations for ABO blood groups and High-quality embryo rate, early abortion rate, birth sex, birth weights and birth defects rate.

As early as 1943, Levine had identified ABO incompatibility as a cause of early abortions and stillbirths. By analyzing the relation of husband/wife joint $A B O$ blood group on 79 couples suffering from recurrent abortion in India, Malekasgar AM et al shows an excess of joint "A/B" blood groups in couples with RSA. (21) However, this study presented the difference simply by the percentage of blood types not $P$ value of statistical analysis. By screening the ABO blood groups aborted fetuses and their parents in 124 early spontaneous abortions, Bandyopadhyay AR found a significantly higher $(P<0.05)$ frequency of $A B O$ incompatibility in couple with miscarriage when compared with newborns and their parents from the same area. ${ }^{(22)}$ This conclusion indicates that the $A B O$ incompatibility between the husband and wife may be a risk factor for early spontaneous abortions. However, we observed lacked statistical power to detect relevant associations of blood groups either to history of spontaneous abortions before ART treatment, nor to early spontaneous abortions following IVF-ET.

Awartani $\mathrm{K}$ et al ${ }^{(10)}$ compared clinical parameters of 566 IVF treatment cycles with different ABO blood type and found no significant association between blood type and pregnancy rate. This result is agreed by another retrospective study which evaluated the effect of non-O blood type on clinical pregnancy of 497 women, and found no statistically significant association between them. As regarding live birth, two retrospective studies on infertile women undergoing IVF came to different conclusions. One study in a cohort of 626 infertile women suggested that woman with blood type B had significantly higher likelihood of live birth following IVF-ET. ${ }^{(12)}$ However, Pereira N et al observed no relation of blood groups to live-birth rate by assessing 2,329 patients undergoing fresh IVF with day 5 single embryo transfer. ${ }^{(13)}$ In our study population, couples who have joint "AB/AB" blood types had significantly higher likelihood of biochemical pregnancy, clinical pregnancy, and live birth. Using the ABO incompatibility effect infertility hypothesize is easy to explain our finding considering same blood type were completely compatible and no antibodies was activated to attack each other.

We found no association between $\mathrm{ABO}$ blood type and birth weight at delivery, which result in the same conclusion with Pereira N. ${ }^{(13)}$ In 1975 Allan TM reported the birth sex ratio of male to female babies is related with ABO blood group of babies and mothers by study 53,679 mother-baby combinations, for AB mothers to have higher sex ratio babies while $A$ babies have lower sex ratio, ${ }^{(24)}$ the authors suggested these differences may cause by interaction of the $\mathrm{ABO}$ genes and some unknown sex-determining genes with estrogen and progesterone. No correlation was found between blood type and birth sex in our cohort.

As mentioned before, the current evidence available is not sufficient to confirm that the blood type ABO is related to some aspects of female fertility. The underlying mechanisms of ABO blood type incompatibility may play some role in abortion are uncovered. Some authors hypothesized that ABO blood type may result in infertility because the presence of incompatible antibodies of spermatozoa in the serum or in the secretions of the wife's genital tract. Genetic is another possible mechanism, given the candidate NR5A1 and TGFBR1 genes impacting oocyte quality or early implantation are proximity to the $9 q 34$ locus of the ABO gene. ${ }^{(25)}$ The other possible mechanism of $A B O-$ related infertility was its effect on thrombosis, some studies suggested the formation of blood vessels at the maternal fetal interface may lead to the failure of implantation or placenta, further influent the clinical outcomes of $\mathrm{IVF}^{(26)}$ the relationship between non-O blood type and venous thromboembolism was observed by previous 
studies, ${ }^{(27)}$ but the data remains controversial. ${ }^{(28)}$ In consideration of maternal-fetal differences in ABO membrane protein structure, studying on couple combinations for ABO blood groups was not sufficient to address the underlying mechanisms. With the limitation of lack of newborn's blood type, it is notable that our results of no association between blood type and birth ratio do not exclude other possibilities. Thus, further prospective studies are needed to prove our results.

\section{Conclusion}

In conclusion, the current large sample size retrospective cohort study demonstrated the statistically significantly positive association between the combination of female blood type $A B$ and male blood type $A B$ with biochemical pregnancy, clinical pregnancy, and live birth rate. Couples who have joint "AB/AB" blood types had significantly higher likelihood of the success rate of IVF/ICSI cycles.

\section{Abbreviations}

ART: Assisted reproductive technology; ICSI: Intracytoplasmic sperm injection; IVF: In vitro fertilization; BMI: Body mass index; OR: Odds ratio; Cl: Confidence interval; COS: Controlled ovarian stimulation; AFC: Antral follicular count; FSH: Follicle-stimulating hormone; LH: Luteinizing hormone; OHSS: Ovarian hyperstimulation syndrome; ET: embryo transfer; FET: Frozen thawed embryo transfer; hCG: Human chorionic gonadotropin; RSA: Recurrent spontaneous abortion

\section{Declarations}

\section{Competing interests}

Authors have declared that no competing interests exist.

\section{Funding}

This work was funded by the International (Regional) Cooperation and Exchange (ICE) Projects of the National Natural Science Foundation of China (NSFC) (FDN- 81820108016 to Yingpu Sun), Joint Construction Project of the Key Project of Medical Science and Technology of Henan Province of China (LHGJ20190121 to Xiao Bao), Projects of the National Science Fund for Young Scholars (82001529 to Feifei Zhao), their support is gratefully acknowledged.

\section{Acknowledgements}

Not applicable.

\section{Authors' contributions}

XB, YPS designed the study; XB, Feifei Zhao and Hao Shi analyzed the data; XB, Zhiqin Bu and Yuling Liang wrote the manuscript; YPS revised the manuscript. All authors contributed to the article and approved the submitted version.

\section{Ethics approval and consent to participate}


This study was approved by the ethics committee of the First Affiliated Hospital of Zhengzhou University.

\section{Consent for publication}

Not applicable.

\section{Availability of data and material}

All data supporting the conclusion of this article are included.

\section{Competing interests}

The authors declare that they have no competing interests.

\section{References}

1. Galliano D. ABO blood group incompatibility and infertility: still an open debate. Fertil Steril. 2017;107(3):579.

2. Behrman SJ, Buettner-Janusch J, Heglar R, Gershowitz H, Tew WL. ABO (H) blood incompatibility as a cause of infertility: a new concept. Am J Obstet Gynecol. 1960;79:847-55.

3. Schwimmer WB, Ustay KA, Behrman SJ. An evaluation of immunologic factors of infertility. Fertil Steril. 1967;18(2):167-80.

4. Solish GI, Gershowitz H. Distribution of ABO blood types among fertile and infertile women. Am J Hum Genet. 1969;21(1):23-35.

5. Nejat EJ, Jindal S, Berger D, Buyuk E, Lalioti M, Pal L. Implications of blood type for ovarian reserve. Hum Reprod. 2011;26(9):2513-7.

6. Lin S, Li R, Chi H, Huang S, Zhang H, Zheng X, et al. Effect of ABO blood type on ovarian reserve in Chinese women. Fertil Steril. 2014;102(6):1729-32 e2.

7. de Mouzon J, Hazout A, Cohen-Bacrie M, Belloc S, Cohen-Bacrie P. Blood type and ovarian reserve. Hum Reprod. 2012;27(5):1544-5; author reply 5-6.

8. Timberlake KS, Foley KL, Hurst BS, Matthews ML, Usadi RS, Marshburn PB. Association of blood type and patient characteristics with ovarian reserve. Fertil Steril. 2013;100(6):1735-9.

9. Pereira N, Hutchinson AP, Bender JL, Lekovich JP, Elias RT, Rosenwaks Z, et al. Is ABO blood type associated with ovarian stimulation response in patients with diminished ovarian reserve? J Assist Reprod Genet. 2015;32(6):985-90.

10. Awartani K, Al Ghabshi R, Al Shankiti H, Al Dossari M, Coskun S. Association of blood groups with ovarian reserve and outcome of in vitro fertilization treatment. Ann Saudi Med. 2016;36(2):116-20.

11. Zhao J, Yao Z, Hao J, Xu B, Wang Y, Li Y. Association of ABO blood groups with ovarian reserve, and outcomes after assisted reproductive technology: systematic review and meta-analyses. Reprod Biol Endocrinol. 2021;19(1):20.

12. Goldsammler M, Jindal SK, Kallen A, Mmbaga N, Pal L. Blood type predicts live birth in the infertile population. J Assist Reprod Genet. 2015;32(4):551-5.

13. Pereira N, Patel HH, Stone LD, Christos PJ, Elias RT, Spandorfer SD, et al. Association between ABO blood type and live-birth outcomes in single-embryo transfer cycles. Fertil Steril. 2017;108(5):791-7. 
14. Matalliotakis I, Cakmak H, Goumenou A, Sifakis S, Ziogos E, Arici A. ABO and Rh blood groups distribution in patients with endometriosis. Arch Gynecol Obstet. 2009;280(6):917-9.

15. Binder H, Flegel WA, Emran J, Muller A, Dittrich R, Beckmann MW, et al. Association of blood group A with early-onset ovarian hyperstimulation syndrome. Transfus Clin Biol. 2008;15(6):395-401.

16. Fang L, He J, Yan Y, Jia Q, Yu Y, Zhang R, et al. Blastocyst-stage embryos provide better frozen-thawed embryo transfer outcomes for young patients with previous fresh embryo transfer failure. Aging (Albany NY). 2020;12(8):6981-9.

17. Hu L, Bu Z, Guo Y, Su Y, Zhai J, Sun Y. Comparison of different ovarian hyperstimulation protocols efficacy in poor ovarian responders according to the Bologna criteria. Int J Clin Exp Med. 2014;7(4):1128-34.

18. Palermo GD, Kocent J, Monahan D, Neri QV, Rosenwaks Z. Treatment of male infertility. Methods Mol Biol. 2014;1154:385-405.

19. Davis OK BP. A textbook of in vitro fertilization and assisted reproduction. New York: The Parthenon Publishing Group. 1999:1- 564.

20. Gardner D SW. In-vitro culture of human blastocysts. Towards Reproductive Certainty Fertility and Genetics Beyond 1999.

21. Malekasgar AM. ABO blood group prevalence in spontaneously repeated abortion. Turk J Haematol. 2004;21(4):181-7.

22. Bandyopadhyay AR, Chatterjee D, Chatterjee M, Ghosh JR. Maternal fetal interaction in the ABO system: $A$ comparative analysis of healthy mother and couples with spontaneous abortion in Bengalee population. Am J Hum Biol. 2011;23(1):76-9.

23. Di Nisio M, Ponzano A, Tiboni GM, Guglielmi MD, Rutjes AWS, Porreca E. Non-O blood group and outcomes of in vitro fertilization. J Assist Reprod Genet. 2018;35(7):1289-94.

24. Allan TM. ABO blood groups and human sex ratio at birth. J Reprod Fertil. 1975;43(2):209-19.

25. Lourenco D, Brauner R, Lin L, De Perdigo A, Weryha G, Muresan M, et al. Mutations in NR5A1 associated with ovarian insufficiency. N Engl J Med. 2009;360(12):1200-10.

26. Hossain N, Paidas MJ. Adverse pregnancy outcome, the uteroplacental interface, and preventive strategies. Semin Perinatol. 2007;31(4):208-12.

27. Franchini M, Mannucci PM. ABO blood group and thrombotic vascular disease. Thromb Haemost. 2014;112(6):1103-9.

28. Di Nisio M, Rutjes AW, Ferrante N, Tiboni GM, Cuccurullo F, Porreca E. Thrombophilia and outcomes of assisted reproduction technologies: a systematic review and meta-analysis. Blood. 2011;118(10):2670-8.

\section{Figures}




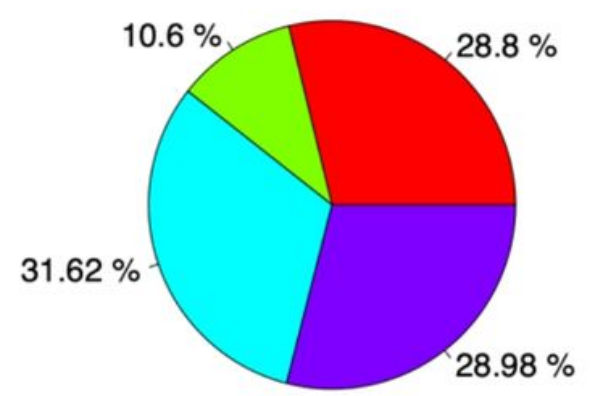

Distribution of Female ABO Blood Type $\%(n=30717)$
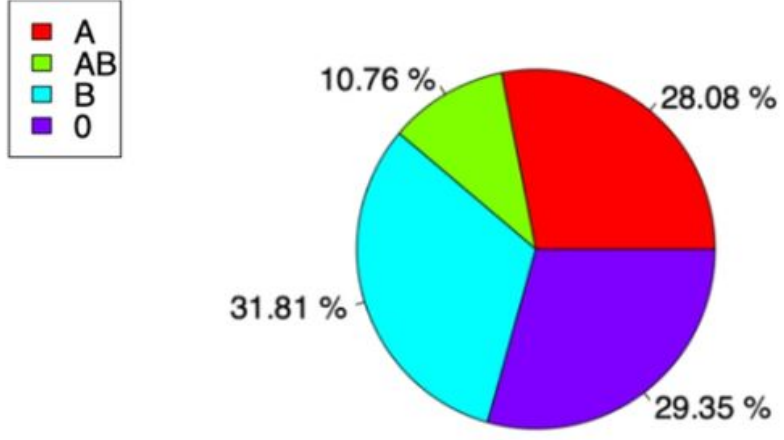

\section{Figure 1}

Distribution of ABO blood type in infertile females and males.

\section{Supplementary Files}

This is a list of supplementary files associated with this preprint. Click to download.

- SupplementaryTables.docx

Distribution of Male ABO Blood Type $\%(n=30717)$ 